\title{
Investigando nuestras propias prácticas: evaluación formativa, TIC y nuevos formatos ${ }^{1}$
}

\author{
Researching our own practice: formative assessment, ICT and new \\ formats
}

Graciela Acerbi, Ana Duboué, Evangelina Méndez y Johana Tagliani²

Fecha de recepción: 23 de marzo de 2020

Fecha de aceptación: 16 de septiembre de 2020

\section{Resumen}

El presente artículo busca contribuir a la reflexión pedagógica, específicamente, a la discusión sobre la evaluación en la formación docente integrando las TIC. Presenta un estudio desarrollado en el marco del Fondo Sectorial de Educación: CFE Investiga, financiado por la ANII (Agencia Nacional de Investigación e Innovación). Se trata de una investigación en educación, que parte de la experiencia en prácticas de evaluación por medio de la realización de cortos, llevada adelante por las docentes involucradas, conjuntamente con la coordinadora de Cineduca del Instituto en el que se desarrolló. El objetivo de la investigación consiste en analizar los efectos que tiene la evaluación formativa a través de la elaboración de productos audiovisuales sobre el aprendizaje de estudiantes de formación docente de un Instituto del interior del país. La metodología aplicada fue de corte cualitativo, resultando un estudio de carácter descriptivo, exploratorio y experimental; las técnicas utilizadas fueron la entrevista, la observación y la revisión documental. Para el análisis de los datos obtenidos, se construyeron categorías conceptuales; en este trabajo, se muestran los resultados de dos de estas: creatividad y trabajo cooperativo, dado que la investigación aún se encuentra en proceso. A nivel de las categorías mencionadas, se observa su resignificación por parte de los estudiantes.

Palabras claves: evaluación; formación docente; TIC; creación audiovisual

\footnotetext{
${ }^{1}$ Investigación desarrollada en el marco del Fondo Sectorial de Educación: ANII-CFE Investiga, cohorte 2018.

${ }^{2}$ Prof. Graciela Acerbi, Docente en IFD de San Ramón e IINN, coordinadora de Cineduca en IFD de San Ramón. Mail: graacerbi@gmail.com.

Mag. Ana Duboué, Docente en IFD de San Ramón e IPA. Mail: aduboue@gmail.com.

Mag. Evangelina Méndez, Docente en IFD de San Ramón e IINN y DOT en IFD de San Ramón. Mail: pedagogias2020@gmail.com

Johana Tagliani, Docente en IFD de San Ramón e IPA, y DOE en IFES. Mail: johanatag@gmail.com.
} 
Acerbi, Duboué, Méndez y Tagliani - Investigando nuestras propias prácticas...

\section{Abstact}

This article aims to contribute to pedagogical thought, specifically to the discussion of evaluation at teacher preparation with the integration of ICTS. This research was developed within the Fondo Sectorial de Educación: CFE Investiga, financed by ANII (Agencia Nacional de Investigación e Innovación). It is an investigation in education, which begins with the experience of evaluation practices through the making of short films, put forward by the teachers involved together with the coordinator of Cineduca, from the institute where it was developed. The objective of the investigation is to analyze the effects that formative evaluation through audiovisual creation has on the learning processes of students from a teacher preparation institute from inland regions of the country. The methodology employed was qualitative, resulting in a descriptive, exploratory and experimental study; the techniques used were interviews, observation and document examination. For the analysis of the obtained data, conceptual categories were constructed; in this work the results of two of these are exposed -as the research is still in progress-: creativity and cooperative work. As for the aforementioned categories, they are observed to be given a new perspective by the students.

Keywords: Evaluation - teacher training - ICT - audiovisual creations

\section{Introducción}

Sin desconocer las problemáticas que atraviesa la práctica evaluativa, e incluso la propia conceptualización de la evaluación, esta investigación asume su naturaleza formativa y la concibe como una poderosa herramienta de aprendizaje.

El universo concreto sobre el cual se indaga es el de la formación docente en el Uruguay y, más específicamente, en grupos de la carrera de Magisterio de un Instituto de formación docente del interior, en la ciudad canaria San Ramón, a $80 \mathrm{~km}$ de la capital del país. La investigación se realiza sobre los efectos de la evaluación formativa por medio de la elaboración de productos audiovisuales, creados por los propios estudiantes de nivel terciario, aunque no universitario.

El Plan Ceibal (2007) y la creación de diferentes programas en el marco del Consejo de Formación en Educación (CFE) han habilitado el uso cotidiano de dispositivos y aplicaciones en las aulas de los diferentes subsistemas educativos nacionales.

Específicamente en los ámbitos del CFE se han generado diferentes espacios de innovación que apuntan al abordaje de las TIC con encuadre pedagógico. Uno de los proyectos surgidos en ese contexto es el Programa Cineduca, que consiste en una propuesta de pedagogía audiovisual creada en 2009, actualmente presente en todos los centros del CFE a través de coordinadores locales. 
Cineduca es una propuesta de naturaleza extracurricular que brinda formación para el abordaje de la cultura y el lenguaje audiovisual desde la apreciación, el análisis y la creación. Cuenta con equipos tecnológicos con nivel de calidad semiprofesional, a disposición de los estudiantes para el rodaje y edición. Una de sus propuestas fundamentales es aprender haciendo. Para ello, realiza instancias de formación y creación, con la intención explícita de que los estudiantes se apropien de los saberes audiovisuales a través del desafío del hacer, se proyecte el interés y se extienda a sus propias prácticas educativas.

El Programa concibe la incorporación del manejo de la tecnología audiovisual como importante herramienta para dar voz a los estudiantes, habilitar sus miradas y propuestas sobre las temáticas tratadas, complejizando su abordaje. No se trata de un mero aprender instrumental, sino que este adquiere sentido si va unido con el para qué y el por qué de tal producción.

En los años de existencia del Programa Cineduca, se han generado diferentes formatos de intervención en cada uno de los centros del CFE, entre los que se encuentra el trabajo interdisciplinario coordinado con docentes de asignaturas curriculares. Estos intercambios se materializan en la creación de productos audiovisuales surgidos a partir de temáticas o conceptos abordados en los cursos. Particularmente la experiencia pedagógica de evaluación desarrollada por un grupo de profesoras de formación docente constituye un ejemplo de ello, lo cual ha derivado en diversas reflexiones profesionales y se ha cristalizado en la investigación que pretendemos compartir a continuación.

Así siendo, este trabajo surge de las experiencias educativas de docentes de Pedagogía, Psicología, Teoría del Conocimiento y Epistemología y Coordinadora de Cineduca del Instituto de San Ramón, que emplean la producción audiovisual creada colectivamente por los estudiantes como la segunda prueba parcial del curso.

Esta investigación ha sido financiada por la ANII en la modalidad Fondo Sectorial de Educación: CFE Investiga-2018 y está siendo asesorada técnicamente y tutorada por el profesor y magíster en Ciencias Sociales y Educación Pedro Ravela. El estudio se ha iniciado en el año 2019, encontrándose, en estos momentos, en la fase de análisis de datos y redacción de resultados.

Por el momento del proceso en que la investigación se encuentra, se presentan sus diversos componentes y se profundiza a nivel de resultados primarios, a partir de dos de las categorías de análisis construidas para reconocer ciertos "saberes» puestos en juego en dichas experiencias de evaluación. Siendo estos el trabajo cooperativo y la creatividad, entendidos como saberes prácticos, en la medida que acompañan y orientan las decisiones y acciones. 
Acerbi, Duboué, Méndez y Tagliani - Investigando nuestras propias prácticas...

\section{Planteamiento del problema de investigación}

\section{La evaluación como problema}

La evaluación es un componente de la práctica educativa que suscita diversas discusiones que van, desde la diferenciación con el mero ejercicio de calificación, hasta fuertes cuestionamientos sobre la imposibilidad de la evaluación de los aprendizajes, en la medida en que ellos son procesos internos en cada sujeto y la pretensión de capturarlos implica la controvertida posibilidad de medirlos y objetivarlos, sin tener en cuenta la modalidad con la que cada sujeto organiza y significa su experiencia. (Santos Guerra, 2017; Pérez Gómez, 2017)

Otros colocan la mirada en el lugar del poder y la autoridad del docente en la evaluación, como es el caso del estudio El ejercicio del poder del maestro en el aula universitaria de Hernández Méndez (2016), el cual plantea una mirada del aula como aquel espacio donde se desarrolla un ejercicio de poder que comprende a educadores y estudiantes, y en el que el primero es el encargado de disciplinar, vigilar y castigar al estudiante. En concomitancia con ello, la evaluación constituiría un recurso didáctico disciplinador, que expone al estudiante y lo premia o castiga.

En este orden de ideas, la investigación El ejercicio del poder docente en la evaluación de los aprendizajes (Pajendino y Piandoy, 2018) concibe la evaluación como una práctica que suele ser carente de sentido para los estudiantes, así como un método de «calificación, clasificación y medio para ejercer el poder» (p. 125) que algunos educadores ven como una herramienta para «medir los conocimientos de los estudiantes» y "controlar los actos de indisciplina en clase» (p.126). Asimismo, agregan que, con el afán de identificar el progreso académico, se ha perdido de vista el sentido de la valoración. Sostienen que es posible que ello se deba al esfuerzo que requiere para el docente otro tipo de abordaje: «observación más detallada y sistemática, a la vez compleja porque posee una connotación más humana, que implica la participación, el trabajo en equipo y las relaciones interpersonales» (p.136).

Los autores concluyen que:

Generalmente, la primera manifestación del poder docente es la autoridad que se ejerce sobre los estudiantes y el proceso evaluativo, ya que socialmente el docente goza de un prestigio, un conocimiento, unas técnicas y unos valores que le dan cierta capacidad de influir sobre los demás (...) En relación al ámbito educativo el poder no se manifiesta exclusivamente mediante la dominación, sino que existen otras manifestaciones que le dan un sentido pedagógico, más humano y productivo al acto de enseñar y al proceso evaluativo. Es en las relaciones entre docente y estudiante, donde subyacen las relaciones de poder, especialmente en el escenario del proceso evaluativo, al ser un proceso continuo 
y permanente que involucra diferentes aspectos que van desde la preparación de clase, el dominio de conocimientos, el manejo de grupo hasta las actitudes y gestos de los docentes. (Pajendino y Piandoy, 2018, p. 135)

En este sentido, esta investigación permite una sistematización de las diversas instancias de evaluación poniendo el foco en el despliegue de ciertas habilidades o saberes de los estudiantes evaluados que trascienden los conocimientos disciplinares. Se concibe la instancia evaluativa como un proceso en el cual el docente asume la autoridad en tanto dirigencia (Cullen, 2000).

\section{Las TIC como oportunidad}

En cuanto a la integración de las TIC en los procesos de enseñanza y aprendizaje, la discusión ya no se dirige a la pertinencia de integrarlas, sino más bien a cómo hacerlo. En este sentido, la evaluación con TIC constituye una oportunidad para producir conocimientos y nuevos recursos haciendo uso del lenguaje digital y audiovisual.

Especialmente para la formación de educadores, el IIPE-UNESCO (2006) sugiere la necesidad de introducir en la formación inicial (de maestros y profesores) enseñanzas que les permitan aprovechar las TIC en sus áreas curriculares y recomienda su continuación en la formación permanente (en Campos y Méndez; 2018, p. 14).

A partir de nuestras experiencias con el uso de TIC, consideramos que puede contribuir a generar conocimiento con sentido para los estudiantes, de forma motivadora, creativa y cooperativa, mediante de la creación audiovisual por parte de estos.

En suma, el estudio busca indagar en los efectos que tiene sobre el aprendizaje de estudiantes de formación docente la evaluación formativa a través de la elaboración de productos audiovisuales. En este sentido, se pretende replicar y validar las experiencias desarrolladas, desde el año 2013, en las propias prácticas pedagógicas de los educadores involucrados.

En lo que refiere a prácticas evaluativas arraigadas, se parte de la constatación de las propias prácticas pedagógicas desarrolladas en diferentes instituciones educativas y del relato de los estudiantes, respecto a la prevalencia de las prácticas memorísticas en la evaluación, las cuales suscitan aprendizajes de tipo superficial, no siendo estos los esperados en ningún nivel educativo, pero menos aún en la formación de los futuros docentes, puesto que son quienes en el posterior ejercicio profesional se hallarán en el lugar clave para perpetuar, o no, tales prácticas. 


\section{Objetivo}

El objetivo general de la investigación que se comparte es el de analizar los efectos que tiene la evaluación formativa a través de la elaboración de producciones audiovisuales en formato documental o ficción sobre el aprendizaje de estudiantes de formación docente de un Instituto del interior del país.

\section{Antecedentes}

Este trabajo fue presentado en el año 2018 como proyecto de investigación. Cuando se realizó el rastreo del estado del arte, no se encontró una investigación que tuviera como objeto de estudio el que específicamente se plantea para este caso: la evaluación formativa en formación docente por medio de la elaboración de audiovisuales. Sin embargo, sí se hallaron varias investigaciones relativas a la evaluación formativa mediante la incorporación de las TIC, así como investigaciones sobre la evaluación formativa en niveles terciarios. Algunas de ellas son las que siguen.

En relación a la evaluación y las diferencias de modelos evaluativos, la investigación de Pelaéz y Osorio (2015) indaga sobre el aprendizaje y la evaluación con prácticas pedagógicas tradicionales e integrando las TIC. Este estudio plantea como uno de sus resultados más sobresalientes que, si bien los desempeños eran igualmente bajos en el pre-test, se constata un sustancial avance en el grupo experimental (con incorporación de TIC) sobre el grupo control.

El artículo La Evaluación Formativa en Docencia Universitaria. Resultados globales de 41 estudios de caso de Vallés Rapp (Universidad de Valladolid), Ureña Ortín (Universidad de Murcia) y Ruiz Lara (Universidad Católica San Antonio de Murcia) presenta los resultados de las prácticas a un año de instalada la Red Nacional de Evaluación Formativa y Docencia Universitaria. Se trata de una interesante fuente para la definición de los criterios que permitirán evidenciar el tipo de aprendizaje que promueve la evaluación formativa.

En el mismo sentido, el estudio Sistemas y procedimientos de Evaluación Formativa en docencia universitaria: resultados de 34 casos aplicados durante el curso académico 2007-2008 de Buscá, Pintor y Martínez (todos del Dpto. Ciencias de la Educación de la Universidad de Oviedo), junto con Peire (Universidad Autónoma de Barcelona), expone las ventajas y debilidades, criterios, procedimientos e instrumentos de la evaluación formativa en la docencia universitaria.

La Organización de Estados Iberoamericanos (OEI), desarrolló conjuntamente con el Consejo de Formación en Educación (CFE) y en colaboración de la Diputación de La Coruña, el Proyecto denominado La práctica pedagógica en entornos innovadores de aprendizaje (2011). Entre las competencias básicas que indican que se deben actualizar para adaptarlas a las evoluciones de la sociedad del conocimiento y la 
información en pro de fortalecer la formación de los docentes de Uruguay, se destaca: establecer lazos de cooperación académica con instituciones extranjeras; ofrecer a los estudiantes de formación en educación la posibilidad de conocer diversos entornos de aprendizaje, así como otras realidades educativas; fomentar el desarrollo de la investigación educativa incorporando enfoques teóricos como culturales; jerarquizar la importancia de la incorporación de entornos de aprendizaje innovadores y creativos que preparen al profesional de la educación para enfrentar una educación para todos en una sociedad cambiante (Demilio, 2011).

La evaluación formativa y participativa es considerada uno de los temas centrales de la docencia universitaria. La Red, que sostiene, implementa y valora estas prácticas de evaluación formativa, posee carácter interuniversitario y su origen se encuentra en 2004-2005 en el Grupo de Evaluación Formativa de la Escuela Universitaria de Magisterio de Segovia. El método que se emplea en esta investigación es el de estudio de caso. También se explicitan algunos requisitos para garantizar una evaluación formativa.

El abordaje de las prácticas de evaluación formativa en la Escuela de Magisterio de Segovia (Universidad de Valladolid) se encuentra disponible la Tesis Doctoral de Hamodi Galán: La evaluación formativa y compartida en educación superior: un estudio de caso, de la Facultad de Educación y Trabajo Social de Valladolid, del año 2014. Este trabajo tiene el doble objetivo de analizar el uso de los sistemas evaluativos de aprendizajes de los estudiantes y conocer la valoración sobre estos sistemas que realizan estudiantes y docentes. Entre sus conclusiones se señalan ventajas e inconvenientes, y la perspectiva de extender internacionalmente la Red de Evaluación Formativa y Compartida en Docencia Universitaria.

Otro estudio, del año 2012, que también analiza la evaluación formativa se titula Análisis de la evaluación formativa en la escuela universitaria de Magisterio de Segovia desde la perspectiva del alumnado, de Marrahí Murillo. En este caso, el estudio fue realizado sobre la muestra de alumnos de la Escuela de Magisterio en la especialidad de Educación Física. Se pone la atención en la relación entre los programas y la evaluación, los instrumentos de evaluación y el desarrollo de competencias consideradas importantes para los estudiantes. Si bien se trata de un trabajo de finalización de grado, es interesante atender uno de los resultados obtenidos en la investigación: concluye que las capacidades que se desarrollan en las asignaturas no son aquellas que los propios estudiantes entienden como importantes en su proceso de formación.

En relación a la producción de cortos como herramienta de evaluación y, por consiguiente, de aprendizaje en profundidad, fuera del país, se encuentran estudios del ámbito de la formación en Comunicación e Información. Es el caso del realizado por Castro Chans, Goñalons, Guastavino Mosna y Sobol (2014): Producción de audiovisuales como estrategia de aprendizaje activo. En este artículo se presenta la 
experiencia en el marco de una asignatura y su análisis crítico. Aunque el objetivo es más específico, ya que se trató de motivar a los estudiantes en la lectura comprensiva de textos, no deja de ser una forma de promover el aprendizaje en profundidad con construcción de conocimiento.

En el artículo Creaciones de Contenidos Audiovisuales producidos por los Estudiantes como nuevo Instrumento en el Proceso de la Enseñanza y Aprendizaje: Metodología y Resultados, de Sánchez Nielsen (2012), se muestran los resultados obtenidos de la actividad de producción de materiales audiovisuales como metodología que fomenta el aprendizaje colaborativo, activo y participativo desde la motivación del estudiante, y agrega, como una fortaleza de la actividad, el interés por el trabajo de otros compañeros.

Es destacable también, como se mencionó anteriormente, la experiencia propuesta y recogida - aunque sin una sistematización rigurosa- que se llevó adelante en el IFD de San Ramón por las profesoras Acerbi, Duboué y Méndez. Se encuentra publicada por la ANEP (2017) en la publicación Sembrando experiencias. Enseñar y aprender en escenarios digitales. Dicho artículo se tituló: Sembrando cortos: evaluación formativa en formación docente.

La producción de material audiovisual durante los años 2016 y 2017 en las asignaturas Pedagogía I, Teoría del Conocimiento y Epistemología (TCE) y Psicología Evolutiva fue elaborada, exhibida y defendida como segunda prueba parcial de los cursos. En la actividad se conjugaron contenidos disciplinares curriculares con los saberes no curriculares aportados por el Programa Cineduca del CFE. Las producciones incursionaron en los formatos ficción y documental. Este tipo de propuestas se viene realizando en el Instituto de Formación Docente desde el año 2013 en la asignatura TCE. Paulatinamente fueron incorporándose otras asignaturas, como Pedagogía I y, actualmente, Psicología Evolutiva. La particularidad de las experiencias del 2017 es que se recogieron posteriormente las valoraciones de los estudiantes que intervinieron, constatándose con ello que esta modalidad de evaluación formativa posee una cantidad considerable de ventajas, en relación a otras formas de evaluar más tradicionales. La valoración positiva es coincidente con la realizada por las docentes involucradas.

\section{Fundamentación teórica}

\section{Interpelando a la evaluación}

La evaluación se integra al proceso de enseñanza y aprendizaje porque ella es un dispositivo para ambos. Como actividad pedagógica, su principal propósito es la mejora del desempeño de estudiantes y docentes, es un instrumento que contribuye a la toma de decisiones y permite la producción de conocimiento. 
La investigación no adhiere a la idea de evaluación sumativa porque su alcance es muy reducido, parte de una concepción positivista del saber y no desarrolla la crítica y autonomía anheladas. En contraposición, se alinea a la noción de evaluación formativa, definida en términos generales como: «Todo proceso de evaluación cuya finalidad principal es mejorar los procesos de enseñanza-aprendizaje que tiene lugar; está orientada a que el alumnado aprenda más y a que el profesorado aprenda a mejorar su práctica docente» (López Pastor, 2012, p.120).

Bajo esta concepción, la noción de evaluación formativa implica que las instancias de evaluación se planifiquen y desarrollen en el propio proceso de enseñanza y aprendizaje de forma motivadora para que los estudiantes pongan en juego diversas estrategias cognitivas, creativas y cooperativas para generar un nuevo conocimiento (López Pastor, 2009).

Una evaluación pensada para este fin busca, en última instancia, que se produzca el aprendizaje profundo. Este, a diferencia del «superficial» o "declarativo», se caracteriza por ser significativo para quien aprende, que tenga sentido para él, implica su comprensión y la posibilidad de dimensionarlo teniendo en cuenta otros conocimientos adquiridos previamente, así como la propia realidad. El estudiante pone en juego "procesos cognitivos complejos» (Ravela, Picaroni y Loureiro, 2019, p.73).

\section{Las TIC y la creación audiovisual en la formación de educadores}

«Sociedad de la información» (Kruger, 2016), «sociedad de redes» (Castells, 2005), «comunidad virtual» (Rodríguez del Rey, 2016) y "cultura de la interacción» (Tapscott, 1997, en Kruger, 2016) son algunas de las acepciones que hacen referencia a las transformaciones que se han llevado a cabo desde el último cuarto del siglo $x x$.

En acuerdo con Levy (2013), consideramos que las TIC constituyen un soporte, y la información debe ser un bien público y su acceso debe estar garantizado (onu, 2001). Para ello, no basta con el uso técnico de las nuevas tecnologías, es menester la adquisición de registros simbólicos, nos referimos a adquisiciones culturales necesarias que aseguren poder el desarrollo de sentidos crítico.

Álvarez y Cantú (2012) advierten que en la apropiación subjetiva singular de la oferta simbólica actual, al incluir y articular procesos caracterizados por la fluidez, la celeridad, la sustitución imaginativa y el cuestionamiento reflexivo promovido por la confrontación con lo diferente, podemos quedar reducidos a meros consumidores de imágenes sin elaboración ni distanciamiento o, por el contrario, enriquecer los modos de representación. Juega así un papel clave el sistema educativo que permite viabilizar, mediante la inclusión de las TIC, el despliegue de los procesos imaginativos, reflexivos y de pensamiento autónomo. 
Quienes llegan a las instituciones educativas no desconocen el uso de la tecnología, cada vez más novedosa y renovada. Las instituciones de educación formal ya no tienen el monopolio de la producción y difusión de significaciones. Sería en vano pedir a los centros educativos que compitan con el poder de seducción e imposición de significaciones que tienen las TIC (Tenti Fanfani, 2006).

Autores como Buckingham (2005) coinciden en que los medios digitales tienen potencial para el aprendizaje, pero no lo explotaremos si continuamos considerándolos como meras tecnologías y no como formas de cultura y comunicación.

Se defiende así el supuesto teórico que el desarrollo de competencias digitales implica el uso crítico y seguro de las tecnologías de la sociedad de la información. Competencias como sistemas complejos de comprensión y de actuación de los individuos en sus interacciones con el escenario en el que habitan, tanto en la vida personal, social, como profesional (Pérez Gómez, 2012).

Aguerrondo (2011) hace referencia a la presencia de un nuevo modelo de conocimiento, donde se agrega la capacidad de acción a las operaciones tradicionales de pensamiento como observar, comparar y razonar. Este nuevo modelo de conocimiento está atravesado por un pensamiento tecnológico que sobrepasa las capacidades informáticas. El sujeto que desarrolle pensamiento y capacidades tecnológicas es capaz de operar sobre la realidad y transformarla, a partir de conceptos y saberes teóricos.

La capacidad de rescatar qué conocimiento necesito para operar de manera innovadora sobre un problema, de ir más allá de lo posible en esas circunstancias, es lo que Aguerrondo denomina creatividad. La hipótesis es que esta creatividad, fundamentalmente cuando se requiere que sea colectiva, no llega automáticamente; esto se dificulta en la medida que la organización de los sistemas educativos, en general, se sustenta sobre un modo de hacer individual, no promoviendo, por lo tanto, el aprender con otros, ni la crítica cruzada, ni el aprendizaje colectivo, por ende, obstruye la creatividad. De esta manera, la autora resalta las posibilidades que brindan las TIC para la innovación y el ejercicio de la creatividad, además de su importante papel en la posibilidad de romper con el elemento estructurante del hacer individual.

Por lo expuesto, ante los desafíos de la formación en educación en el siglo xxI, es preciso abocarse al logro de una formación profesional y profesionalizante, integral, apuntando a la promoción de idoneidad académica, así como competencias ciudadanas que permitan pensar, decidir y hacer responsablemente, establecer una mirada crítica sobre los fenómenos actuales, tomar decisiones informados, participar, argumentar y crear. 
Acerbi, Duboué, Méndez y Tagliani - Investigando nuestras propias prácticas...

\section{El audiovisual y la evaluación formativa en la formación docente}

La cultura y el lenguaje audiovisual, en la realidad contemporánea, constituyen parte innegable de nuestro universo cultura de referencia. En palabras de Inés Dussel (2006):

La forma que tenemos de pensarnos a nosotros mismos, y de pensar a los demás, se da en el marco de estereotipos, formas, íconos, que nos vienen provistos por esta cultura en la que vivimos. Negarla, hacer de cuenta que no existe, no sirve, ni contribuye a que lo que transmitamos como educadores sea relevante y productivo para aquellos a quienes estamos educando. (p. 284)

Pensar el aula como un espacio para la creación audiovisual implica una resignificación de la relación entre los alumnos. La realización de un trabajo colectivo en que se embarcan para que se logre un producto que los refleje a todos y por el que todos van a ser valorados.

El producto de su trabajo no finaliza con la aprobación de un curso, se proyecta en un audiovisual que seguirá circulando, generando repercusiones, dialogando con los espectadores. La evaluación se redimensiona en un objeto concreto que permanece y habla de ellos como protagonistas de un proceso de creación en donde se ponen en juego saberes y decisiones técnicas, estéticas y éticas.

Entendemos que la experiencia de evaluar la evaluación consiste en una práctica pedagógica profundamente formativa en este nivel educativo, por lo cual contribuye al aprendizaje de la profesión de educador. Asimismo, la evaluación entendida como una parte constitutiva de las prácticas pedagógicas que desarrollan los docentes requiere de constante reflexión y puesta en tensión para generar nuevas estrategias que posibiliten, no solo la acreditación o demostración de un saber determinado, sino también y sobre todo constituya una instancia de aprendizaje.

En este orden de ideas, el proceso de creación de producciones audiovisuales sobre temáticas o conceptos disciplinares pone en juego una serie de procesos, metodologías de trabajo, apropiación y circulación de saberes que reúne las características que lo constituyen en un caso de evaluación formativa auténtica. La que surge como práctica alternativa a aquellas formas de evaluar, memorísticas y carentes de sentido, en tanto declarativas, que buscaban la reproducción por medio de repetición rutinaria de ejercicios (Ravela, Picaroni y Loureiro, 2017, p. 96).

Entendemos la íntima relación entre la evaluación auténtica con un aprendizaje de igual característica, puesto que se trata de una evaluación formativa, integrada al proceso de aprendizaje. Según Wiggins (en Ravela et al, 2019, p. 97), «El logro intelectual auténtico está basado en tres criterios: la construcción de conocimientos 
Acerbi, Duboué, Méndez y Tagliani - Investigando nuestras propias prácticas...

por parte del estudiante (1), a través de la indagación disciplinar (2) que tiene valor más allá del salón de clase».

\section{Diseño y metodología}

El estudio busca analizar los efectos que tiene sobre el aprendizaje de estudiantes de formación docente la evaluación formativa a través de la elaboración de producciones audiovisuales en formato documental o ficción. Para ello, se propone una metodología cualitativa, de corte exploratorio, descriptivo y experimental.

Se opta por trabajar con una muestra intencionada y no probabilística. Dicha muestra está constituida por dos grupos de Magisterio de primero y segundo año, de las asignaturas Pedagogía y Teoría del Conocimiento y Epistemología, respectivamente (grupos de intervención). En dichos grupos se desarrollan las prácticas de evaluación formativa mediante la producción de audiovisuales. Asimismo, se cuenta con dos grupos control en el mismo Instituto, en las mismas asignaturas y grados en los que se lleva a cabo una evaluación en formato de producción escrita, con el objetivo de comparar el tipo de aprendizaje que se genera en los grupos experimentales y contrastarlos con los grupos de control.

Para que se pudiese definir las características de cada uno de los grupos al comienzo del año lectivo, se realizó un pretest a los cuatro grupos, el cual permitió reconocer edades, recorridos académicos previos, percepciones sobre la formación docente y los modos de evaluar, así como de experiencias de evaluación anteriores.

Las técnicas de recolección de datos fueron: la encuesta a la totalidad de los alumnos (anónima), la entrevista semiestructurada cuya finalidad fue profundizar lo obtenido por la aplicación de la encuesta y, por último, la técnica de la observación que permitió recabar datos sobre las prácticas desarrolladas por las educadoras. Todas las instancias de evaluación fueron acompañadas de una posterior autoevaluación y evaluación de las evaluaciones por parte de los estudiantes, lo que constituyó otro insumo para la observación.

En los cuatro grupos se implementó estrategias de enseñanza tendientes a fomentar y potenciar fundamentalmente saberes correspondientes a estas dos categorías que remiten a los saberes prácticos: trabajo cooperativo y creatividad. Desde las asignaturas Pedagogía y Teoría del Conocimiento Epistemología se planificaron y plantearon actividades, desafíos y problemas que requerían resoluciones desde la exploración y divergencia, así como la organización y el sentido de trabajar con otros en un fin común. Sin embargo, solamente en dos de los grupos, los grupos de intervención (un primero y un segundo), se propuso como actividad de evaluación la elaboración de un corto. 
El análisis de datos y las comparaciones que se hicieron —en una primera etapa, fue de cada grupo en sí mismos y, luego, entre los grupos de intervención con los de control- tuvieron como eje una serie de categorías generadas por el equipo investigador. Estas contienen saberes que, a nuestro juicio, constituyen los procesos que favorecen un aprendizaje profundo, por lo que funcionan como criterios del mismo. Además de los ya mencionados (trabajo cooperativo y creatividad), se incluyen: sentido, metacognición, motivación y autonomía. Consideramos que cada categoría supone saberes determinados que resultan pasibles de visualizar en los desempeños o en las producciones de los estudiantes a partir de la definición de un grupo de descriptores para cada uno de ellos.

En el punto de partida, los cuatro grupos no presentan diferencias sustanciales. Las edades de los estudiantes oscilan entre los 18 y 42 años, con trayectorias educativas diversas, pero en planes donde las evaluaciones consistían básicamente en el tradicional escrito de preguntas y respuestas.

Las diferencias de resultados que se obtuvieron a partir de la aplicación de las entrevistas y observaciones iniciales entre los grupos de intervención y los grupos testigo, previo al desarrollo de la evaluación formativa con producción de recursos audiovisuales, no fueron significativas. Es preciso aclarar que estas técnicas se aplicaron en la etapa en que las actividades de evaluación para todos los grupos eran de igual naturaleza: pruebas escritas individuales.

Sin embargo, las observaciones realizadas en el transcurso de los segundos parciales, momento en el cual se presenta la diferencia en las evaluaciones, evidencian que, en los grupos donde se realizó la producción audiovisual, se generaron otras formas de organizarse, así como también se dieron cambios en la percepción de la propia evaluación. De las entrevistas se obtiene que los grupos testigos continúan sosteniendo la incomodidad frente a la instancia de evaluación (prueba parcial subgrupal escrita), mientras que los grupos de intervención manifiestan un cambio, valorando positivamente la modalidad de evaluación (prueba parcial subgrupal con producción audiovisual).

\section{Resultados}

Si bien la investigación se encuentra en la primera etapa de análisis de datos, se puede enunciar algunos primeros hallazgos, tales como que dos categorías definidas para el aprendizaje profundo se vieron más potenciadas y desarrolladas mediante el uso de las tecnologías, a saber: trabajo cooperativo y creatividad

En primer lugar, definimos el trabajo cooperativo como el conjunto de acciones desarrolladas en grupos, con el cometido de resolver un problema y promover el aprendizaje individual y colectivo; permite romper con la cultura de la competitividad 
y afianzar el trabajo grupal mejorando los aprendizajes de todos. (Johnson, Johnson \& Holubec, 1999; Vilches y Gil Pérez, 2012)

En ese sentido, se pudo constatar que los estudiantes de los cuatro grupos contaban con algunas estrategias para el trabajo cooperativo al inicio del año. Expresaron una alta valoración del mismo, pero vinculada con la optimización del tiempo y con la comprensión de los textos.

Con posterioridad a la experiencia de evaluación formativa con audiovisual, se logró identificar el desarrollo de diversas habilidades como la tolerancia, el respeto y la empatía, las cuales no se encontraban enunciadas por los estudiantes previo a esta experiencia de evaluación, pese a que habían sido parte de actividades grupales. Es decir, los estudiantes lograron identificar el desarrollo de estas habilidades a partir de la experiencia de evaluación mediante la producción de recursos audiovisuales.

Asimismo, se puede constatar una diferencia cualitativa en relación a la concepción del trabajo cooperativo y su relevancia. Mientras que, al inicio, como se expuso, se le otorga un valor de tipo instrumental, en el segundo parcial los grupos de intervención manifiestan que el trabajo cooperativo es enriquecedor a nivel personal; sin embargo, los grupos testigo no modifican su percepción.

En la observación, se ha podido constatar un mayor disfrute y compromiso en aquellos grupos en donde la evaluación ha sido la producción audiovisual, en tanto en las modalidades más tradicionales se percibió altos niveles de ansiedad.

En lo que respecta a la categoría de creatividad, la concebimos como la generación y expresión de nuevas ideas, valores y significados de manera singular en los campos del conocimiento abordados, considerando sus implicancias éticas. Esta categoría que toma en cuenta para su formulación la caracterización que propone López Calichs (2016) en la que la fluidez, la iniciativa y la elaboración de respuestas alternativas, poniendo en juego conocimientos para resolver un problema (Aguerrondo, 2010), son sus dimensiones constitutivas. Esta concepción se nutre con en el análisis de proceso creador que propone Augustowsky (2017):

Al iniciar un proceso creador, se pone en juego la capacidad de proyectar, imaginar, valorar, revisar opciones que activan componentes de orden racional, ligados al pensamiento científico, pero también de orden emocional. (p.86)

En este sentido se evidencia, a través de las actividades cuyas propuestas incluyen libertad para resignificar y plantear nuevas maneras de concebir o problematizar situaciones, que los grupos de los mismos niveles (primero y segundo) partieron de realidades similares. Los grupos de primer año se mantuvieron atados a las consignas, buscando el aval permanente del docente, no quedando claro para los estudiantes cuán posible es apropiarse de una actividad de esas características. 
Los grupos de segundo año demostraron un potencial manifiesto frente a la posibilidad de generar nuevas formas de concebir y utilizar una idea inicial, manejando, en general, un interesante nivel simbólico. Esto quedó más evidenciado en las actividades iniciales realizadas por el grupo testigo que por el grupo que realizaría el audiovisual.

Al final del proceso, en las entrevistas y en los trabajos autoevaluativos, los estudiantes del grupo de primer año que realizaron el cortometraje de ficción demostraron haber pasado por una experiencia en la que se puso en juego la creatividad. El espacio para proponer posibles historias, el surgimiento de ideas heterogéneas para dialogar entre los conceptos y el relato audiovisual, los aprendizajes tecnológicos desarrollados y puestos en juego para plasmar las ideas y el logro de filmar y editar la ficción estuvieron presentes y fueron valorados de forma entusiasta. Todo ello es reconocido por los estudiantes involucrados como un aprendizaje y como una fortaleza que pautó las características de la producción.

Estas percepciones se repiten en menor medida en el grupo que se abocó a la realización del documental, ya que, si bien no nombraron la creatividad como una de las características a resaltar en su trabajo final, valoraron las instancias que ponen en juego esta dimensión como importantes en la resignificación de la experiencia de ser evaluado. En ese sentido, explicitan y valoran la posibilidad de mirar la teoría desde otra perspectiva, los espacios para proponer, las posibilidades de vincular lo teórico con realidades concretas que pueden asir y revisar a partir del registro audiovisual realizado. Estiman valiosa la posibilidad de construir sus interpretaciones, colectivizadas en el proceso creativo que se desarrolla en la edición del registro audiovisual, elaborando un relato propio.

Por contraparte, entre los integrantes de los grupos que no realizaron el audiovisual como parcial, ya sea en el grupo de primer año y como en el de segundo, aparece la palabra creativa para valorar la propuesta escrita tradicional, sin argumentos ni explicaciones que permitan constatar la presencia de instancias creativas experimentadas por los estudiantes.

Tomando en cuenta las situaciones al inicio de los cuatro grupos y los procesos desarrollados, tal como son valorados por los estudiantes, se puede inferir que el desarrollo de la creatividad se propicia en los grupos en la medida en que las propuestas de aula habiliten un clima pedagógico creativo (López Calchis, 2016) y no se restrinja a través pautas marcadas por recorridos fijos.

Por otra parte, trascendiendo a los hallazgos comentados, se constata la emergencia de un elemento novedoso: la transformación, en los estudiantes, de la concepción de la evaluación en sí misma. 


\section{Conclusiones}

El estudio, que se encuentra en fase de redacción de los primeros resultados, constituye un aporte relevante en la medida que, entre otros aspectos, aporta conocimiento en un área poco explorada y vertebral en la formación docente: la evaluación de aprendizajes.

Asimismo, resulta preciso subrayar que este estudio es producto de la reflexión sistemática sobre las propias prácticas pedagógicas, lo cual constituye un impulso a los diversos profesionales de la educación para que continúen nutriendo el campo pedagógico y didáctico a partir de la investigación en educación. Por otro lado, contribuye al campo de las Tecnologías Educativas en la medida que la experiencia implica la integración de las TIC en la formación docente.

Tomando en cuenta las situaciones de inicio de los cuatro grupos y los procesos desarrollados a través de las valoraciones de los estudiantes, se puede establecer que, además de los conocimientos académicos propios de cada asignatura, se pueden desarrollar otros saberes en los procesos de evaluación, siempre y cuando las propuestas de aula y las evaluaciones sean formativas y no los restrinjan a través pautas marcadas.

En este sentido, se puede afirmar que los saberes como trabajo cooperativo y creatividad, se encuentran habilitados por propuestas desarrolladas por sujetos (docentes y estudiantes) que colocan el pensamiento y las capacidades tecnológicas como un elemento constitutivo de sus prácticas. Estas ponen en juego las TIC, en tanto elemento pedagógico que permite llevar a cabo procesos de aprendizaje profundo. Los grupos implicados en la creación audiovisual reconocen haber vivido un cambio cualitativo en sus experiencias de cooperación, por tanto, en la concepción de la misma.

Con respecto a la creatividad, ambos grupos se apropian de los espacios que la propuesta docente instala, reconociéndolos como ámbitos para imaginar, proyectar ideas propias y revisar en colectivo vinculaciones a las referencias teóricas de origen, con el objetivo de llevar a cabo la creación audiovisual. Ponen en juego, para ello, las capacidades necesarias y las formas tecnológicas de pensar que se promueven con la creación audiovisual. Reconocen las experiencias de evaluación vividas como instancias positivas en donde la incertidumbre y los desafíos son valorados en tanto elementos enriquecedores de sus aprendizajes teóricos, así como de su formación como futuros evaluadores, tanto a nivel individual como grupal.

Fundamentalmente el cambio que se observó fue de naturaleza cualitativa, en la medida en que los cuatro grupos reconocen el valor del trabajo cooperativo y se observa cierto grado de creatividad, pero en los grupos de intervención se 
transforma y enriquece el sentido de estos. Los estudiantes que protagonizaron la propuesta de creación audiovisual reconocen la posibilidad de creación en la cooperación.

Finalmente, podemos afirmar que los estudiantes problematizan la propia evaluación, lo que constituye un aprendizaje significativo en sujetos que harán de esta una de sus principales prácticas profesionales e incluyen en ella las TIC como instrumento pedagógico para un aprendizaje en profundidad.

\section{Bibliografía}

Acerbi, G. Duboué, A. y Méndez, E. (2017). Sembrando cortos: evaluación formativa en formación docente experiencias. En: Quinteros, M. (Comp.) Sembrando experiencias. Enseñar y aprender en escenarios digitales. (pp. 45-54). Montevideo, Uruguay: ANEP.

Aguerrondo, I. (2011). Estímulos organizacionales para el pensamiento innovador. En: Seminario Internacional La práctica pedagógica en entornos innovadores de aprendizaje. (pp. 43-52). Montevideo, Uruguay: ANEP - CODICEN

Álvarez P. Cantú G. (2012). Adolescentes y nuevas tecnologías: nuevas aproximaciones a los procesos psíquicos implicados. IV Congreso Internacional de Investigación y Práctica Profesional en Psicologían XIX Jornadas de Investigaciónn VIII Encuentro de Investigadores en Psicología del MERcosUR. Facultad de Psicología - Universidad de Buenos Aires. Recuperado de: https://www.aacademica.org/000-072/417

Augustowsky, G. (2017). La creación audiovisual en la infancia. De espectadores a productores. Buenos Aires, Argentina: Paidós.

Buckingham D. (2005). Educación en medios. Alfabetización, aprendizaje y cultura contemporánea. Recuperado de: http://www.terras.edu.ar/biblioteca/2/2BUCKINGHAM-David-Alfabetizaciones-Digit ales.pdf

Buscá, F., Pintor, P., Martínez, L., y Peire, T. (2016). Sistemas y procedimientos de Evaluación Formativa en docencia universitaria: resultados de 34 casos aplicados durante el curso académico 2007-2008. Estudios sobre Educación, 18, 255-276 Recuperado https://www.unav.edu/publicaciones/revistas/index.php/estudios-sobre-educacion/ article/view/4674.

Campos, N. y Méndez, E (2018). Competencias Digitales Docentes para integrar las Tecnologías de la Información y la Comunicación a las prácticas pedagógicas de formación docente. Tesis inédita de Maestría. Claeh, Uruguay.

Castells M. (2005). La Era de la Información Vol. ı: La Sociedad Red. Madrid, España: Alianza Editorial.

Castro Chans, B., Goñalons, G., Guastavino Mosna, M. L., \& Sobol, B. (2014). Producción de audiovisuales como estrategia de aprendizaje activo. En: IX 
Congreso sobre Tecnología en Educación \& Educación en Tecnología (La Rioja, 2014), pp. 332-341. Recuperado de: http://sedici.unlp.edu.ar/handle/10915/38646

Demilio, L. (2011). Prólogo. En: Seminario Internacional La práctica pedagógica en entornos innovadores de aprendizaje. (p. 7). Montevideo, Uruguay: ANEP - CODICEN

Dussel, I. Gutierrez, D. (Comp.). (2006). Educar la mirada: políticas y pedagogías de la imagen. Buenos Aires, Argentina: Ed. Manantial.

Cullen, C. (2000). Crítica de las razones de educar. Temas de filosofía de la educación. Buenos Aires, Uruguay: Paidós.

Guerra, M. Á. S. (2003). Una flecha en la diana: La evaluación como aprendizaje (Vol. 166). Madrid, España: Narcea Ediciones.

Hamodi Galán, C. (2014). La evaluación formativa y compartida en educación superior: un estudio de caso. Tesis doctorales UVa. Universidad de Valladolid. Facultad de Educación y Trabajo Social. Recuperado de: http://uvadoc.uva.es/handle/10324/5668

Hernández Méndez, G. (2006). El ejercicio del poder del maestro en el aula universitaria. CPU-e, Revista de Investigación Educativa, (2), pp. 1-17. Veracruz, México: Instituto de Investigaciones en Educación. Recuperado de: https://www.redalyc.org/pdf/2831/283121711005.pdf

Johnson, D. W., Johnson, R. T., \& Holubec, E. J. (1999). El aprendizaje cooperativo en el aula. Buenos Aires, Argentina: Paidós. Recuperado de: https://www.guao.org/sites/default/files/biblioteca/El\%20aprendizaje\%20cooperati vo\%20en\%20el\%20aula.pdf

Kruger, K. (2006). El Concepto de Sociedad del Conocimiento. Revista Bibliográfica de Geografía y Ciencias Sociales, $x(683)$. Recuperado de: http://www.ub.edu/geocrit/b3w-683.htm

Levy D., Rasocovan S., Karienfeld D. (2013). Entre adolescentes y adultos en la escuela. Buenos Aires, Argentina: Paidós.

López Calichs,E. (2006). El proceso de formación de las competencias creativas. Una necesidad para hacer más eficiente el aprendizaje de los estudiantes universitarios. Revista Iberoamericana de Educación 40(3). Recuperado de: https://www.researchgate.net/publication/28125086_El_proceso_de_formacion_d e_las_competencias_creativas_Una_necesidad_para_hacer_mas_eficiente_el_a prendizaje_de_los_estudiantes_universitarios

López Pastor, V. M. (2012). Evaluación formativa y compartida en la universidad: clarificación de conceptos y propuestas de intervención desde la Red Interuniversitaria de Evaluación Formativa. Psychology, Society \& Education, 4(1), pp. 117-130.

López Pastor, V. M. L. (2009). Evaluación formativa y compartida en Educación Superior: propuestas, técnicas, instrumentos y experiencias. Madrid, España: Narcea Ediciones.

Martínez Alvarado, H. (2011). Docentes del siglo xxl: Innovar o innovar. En: Seminario Internacional La práctica pedagógica en entornos innovadores de aprendizaje. (pp. 27-32). Montevideo, Uruguay: ANEP - CODICEN 
Acerbi, Duboué, Méndez y Tagliani - Investigando nuestras propias prácticas...

Peláez, L. E., Osorio, B. E. (2015). Medición del nivel de aprendizaje con dos escenarios de formación: uno tradicional y otro con TIC. Entre Ciencia e Ingeniería. Recuperado de: https://pdfs.semanticolarsch.org/79f1/293df4d7426b159576683131d43ae3e82284 .pdf

Pérez Gómez, Á. I. (2012). Educarse en la era digital. Madrid, España: Morata.

Pérez Gómez, Á. I. (2017). Pedagogías para tiempos de perplejidad. Santa Fe, Argentina: Homosapiens.

Piandoy, O. E. P. y Jojoa, R. H. P. (2015). La evaluación como ejercicio del poder docente. Revista Historia de la Educación Colombiana, 18(18), pp. 121-142. Recuperado de: https://revistas.udenar.edu.co/index.php/rhec/article/view/4108

Rapp, C. V., Ortín, N. U., y Lara, E. R. (2011). La evaluación formativa en docencia universitaria: resultados globales de 41 estudios de caso. REDU: Revista de Docencia Universitaria, 9(1), pp. 135-158. Recuperado de: https://polipapers.upv.es/index.php/REDU/article/view/6184/6234

Ravela, P., Picaroni, B. y Loureiro, G. (2019). ¿Cómo mejorar la evaluación en el aula? Reflexiones y propuestas de trabajo para docentes. Montevideo, Uruguay: Magró.

Rodriguez del Rey, L.; Rodriguez del Rey Rodriguez, M. y Perez Fleites, M. (2016). Las comunidades virtuales de aprendizaje: sus orígenes. Universidad y Sociedad, 8(4), pp. 93-101. Recuperado de:

http://scielo.sld.cu/scielo.php?script=sci_abstract\&pid=S2218-36202016000400012

Sánchez Nielsen, E. (2012). Creaciones de contenidos audiovisuales producidos por los estudiantes como nuevo instrumento en el proceso de la enseñanza y aprendizaje: metodología y resultados. Jornadas de Enseñanza de la Informática, pp. 223-230. Recuperado de: https://upcommons.upc.edu/handle/2099/15051.

Santos Guerra, M. Á. (2017). Evaluar con el corazón. Santa Fe, Argentina. Homosapiens.

Sutz, J. (2011). O inventamos o erramos. Revisando las fuentes de la innovación en el siglo XXI. En: Seminario Internacional La práctica pedagógica en entornos innovadores de aprendizaje. (pp. 19-26). Montevideo, Uruguay: ANEP - CODICEN

Tenti Fanfani, E. (2017) La escuela y la visión no alfabética. La Tercera Fase. Formas de saber que estamos perdiendo. En: Sitio FLACSO Virtual. Curso: Educación, imágenes y medios. Cohorte 12. Clase 2.

Vilches, A. y Gil Pérez, D. (2012). El trabajo cooperativo en el aula. Una estrategia considerada imprescindible pero infrautilizada. Aula de Innovación Educativa, 208, pp. 41-46. 